\title{
Bcl-2 Associated Athanogene 2 (BAG2) is Associated With Progression and Prognosis of Hepatocellular Carcinoma: A Bioinformatics-Based Analysis
}

\author{
Xi Zhang ${ }^{1}$, Junjun Zhang ${ }^{1}$, Yang $L i u^{2}$, Jie $L i^{3}$, Juan $\operatorname{Tan}^{2}$ and Zewen Song ${ }^{1 *}$ \\ ${ }^{1}$ Department of Oncology, The Third Xiangya Hospital of Central South University, Changsha, China, ${ }^{2}$ Department of Pathology, \\ The Third Xiangya Hospital of Central South University, Changsha, China, ${ }^{3}$ Department of Information Science and Engineering, \\ Hunan University of Chinese Medicine, Changsha, China
}

Background: $\mathrm{Bcl}-2$ associated athanogene2 (BAG2) is reported to act as an oncogene or a tumor-suppressor in tumors in a context-dependent way; however, its function in hepatocellular carcinoma (HCC) remains unclear.

Methods: Immunohistochemistry $(\mathrm{IHC})$ staining, cell counting kit-8 (CCK-8) assay, apoptotic assay, cell invasion assay and a set of bioinformatics tools were integrated to analyze the role of BAG2 in hepatocellular carcinoma.

Results: BAG2 was significantly up-regulated in HCC. Prognostic analysis indicated that HCC patients with high expression of BAG2 had significantly shorter overall survival, progression free survival and disease specific survival. Besides, silencing BAG2 in HCC cells impaired cell proliferation, facilitated apoptosis and repressed invasion of the cells. Bioinformatics analysis showed that BAG2 might regulate ribosome biogenesis in HCC.

OPEN ACCESS

Edited by: József Tímár,

Semmelweis University, Hungary

*Correspondence:

Zewen Song

xy3szw@163.com

Received: 13 August 2020 Accepted: 12 February 2021

Published: 01 April 2021

Citation:

Zhang X, Zhang J, Liu Y, Li J, Tan J and Song Z (2021) BCl-2 Associated Athanogene 2 (BAG2) is Associated With Progression and Prognosis of Hepatocellular Carcinoma: A Bioinformatics-Based Analysis. Pathol. Oncol. Res. 27:594649. doi: 10.3389/pore.2021.594649
Conclusion: This study revealed that the up-regulated BAG2 in HCC was associated with a worse prognosis and might favor the progression of the disease.

Keywords: BAG2, hepatocellar carcinoma, ribosome biogenesis, invasion, apoptosis

\section{INTRODUCTION}

Hyperactive ribosome biogenesis is a hallmark of many types of cancer including hepatocellular carcinoma (HCC) [1, 2]. Undoubtedly, increased ribosome biogenesis and protein synthesis is essential to support tumor proliferation and growth, and some recent studies indicate that aberrant regulation of ribosomes could drive tumorigenesis [1]. In addition, ribosome biogenesis is further found to be required for the epithelial-mesenchymal transition (EMT) of cancer cells during tumor invasion [3]. CX-5461, the first-in-class selective ribosome DNA (rDNA) transcription inhibitor, demonstrates anti-tumor activity against advanced hematologic cancers, with the best response being partial response (PR) sustained for more than 12 months [4]. Thus, targeting components or regulators of ribosome biogenesis represents a new therapeutic strategy in cancer treatment.

The Bcl-2 associated athanogene (BAG) family, first known as a group of proteins preventing cell death through their interaction with $\mathrm{Bcl}-2$, participates in the regulation of physiological activities ranging from apoptosis to tumorigenesis $[5,6]$. To date, six members of the family have been 
identified in humans and are named from BAG1 to BAG6. The function of these proteins have been well studied in neurodegenerative disorders like Alzheimer's and Parkinson's disease, but recently the family has become a focus in canceroriented research [5]. BAG2 shares a C-terminal conserved region (the BAG domain) with other members of the family, allowing it to bind the HSP70 family of molecular chaperones [5]. As a member of the BAG family, BAG2 is also found to play an essential role in several types of cancer [7-10]. Kyung-Min Yang et al. found that BAG2 was significantly over-expressed in triplenegative breast cancer (TNBC) and favored tumor progression by regulating pro-cathepsin $\mathrm{B} /$ annexin II complex formation and facilitating the secretion of pro-cathepsin $\mathrm{B}$, which induces metastasis [9]. Besides, elevated level of BAG2 in tumors might cause accumulation of mutant p53 proteins that drive tumor growth, by binding to the proteins and inhibiting MDM2mediated ubiquitination and degradation [8]. An oncogenic function of BAG2 is also reported in gastric cancer and oral cancer $[7,10]$. However, BAG2 might play a pro-apoptotic role in thyroid carcinoma, because silencing the expression of the gene partially inhibits cell death in these cells exposed to the proteasome inhibitor MG132 [11]. This inconsistency indicates that BAG2 might function as an oncogene or a tumor-suppressor in tumors in a context-dependent way.

Since no study, to our knowledge, explores the function of BAG2 in HCC, it is unclear whether the gene plays a role in the disease. In this study, we found that BAG2 was significantly upregulated in HCC, and patients with high expression of BAG2 showed a significantly shorter overall survival (OS), progression free survival (PFS) and disease specific survival (DSS). In vitro experiments showed that silencing BAG2 in HCC cells impaired cell proliferation, facilitated apoptosis and repressed invasion of the cells. By using a set of bioinformatics tools, we further found that BAG2 might participate in the ribosome biogenesis of HCC.

\section{MATERIALS AND METHODS}

\section{Cell Culture and Human Tissue}

HepG2 cells were obtained from the Chinese Academy of Sciences Cell Bank (Shanghai, China), cultured in RPMI-1640 medium (Invitrogen, United States) supplemented with 10\% fetal bovine serum (FBS, ExCell, China), and were incubated under humidified atmospheric conditions with $5 \% \mathrm{CO}_{2}$ at $37^{\circ} \mathrm{C}$ [12]. HCC patients' tumor $(n=10)$ and tumor-adjacent normal tissues $(n=10)$ were collected in the Third Xiangya Hospital and used for immunohistochemistry (IHC) staining of BAG2. The clinicopathological characteristics of these patients were summarized in Supplementary Table S1. Use of human tissue was approved by the institutional review board (IRB) of the Third Xiangya Hospital, Central South University (No: 2020-S374). Written informed consent form (ICF) had been obtained from patients whose tissues were used in this study.

\section{RNA Extraction and RT-PCR}

Total RNA was extracted by using Trizol (Pufei Biotech Co., Ltd., Shanghai, China), and then quantified with the Nanodrop 1,000
(Thermo Fisher Scientific, United States), followed by cDNA synthesis (Promega, United States). 6 ul SYBR Premix Ex Taq (TAKARA, Japan), 0.3 ul primers, 0.6 ul cDNA, and 5.1 ul RNasefree $\mathrm{H}_{2} \mathrm{O}$ were added into 96-well plates for Real-time PCR (RTPCR) on the LightCycler 480 II (Roche, Switzerland). The running parameters were set as: $95^{\circ} \mathrm{C}$ for $30 \mathrm{~s}, 40$ cycles of PCR $\left(95^{\circ} \mathrm{C}\right.$ for $5 \mathrm{~s}$ and $60^{\circ} \mathrm{C}$ for $\left.30 \mathrm{~s}\right)$, and dissociation $\left(95^{\circ} \mathrm{C}\right.$ for $15 \mathrm{~s}, 60^{\circ} \mathrm{C}$ for $30 \mathrm{~s}$ and $95^{\circ} \mathrm{C}$ for $15 \mathrm{~s}$ ) [13]. The primers used in this work were obtained from Ruan Tuo Biotechnologies (Shanghai, China) and their sequences were included in Supplementary Table S2. Results were first normalized against the housekeeping gene GAPDH, which is stable across all samples, and then against the experimental controls.

\section{Small Interfering RNA Silencing of BAG2}

Three small interfering RNAs targeting BAG2 (siBAG2), named from si-BAG2-1 to si-BAG2-3, and a negative control (NC) siRNA (si-NC) were designed by and purchased from Ruan Tuo Biotechnologies (Shanghai, China). The sequences were si-BAG2-1 (sense : $5^{\prime}$-GUACUAGGAUCUAGCAUAUUUTT3', Anti-sense : 5'-AAAUAUGCUAGAUCCUAGUACTT-3'), si-BAG2-2 (sense : $5^{\prime}$-GAUC-AGAAGUUUCAAUCCAUATT3', Anti-sense : 5' - UAUGGAUUGAAACUUCUGAUCTT-3'), and si-BAG2-3 (sense : $5^{\prime}$ - AGCAUGCCACAAGGAUUAUUG TT-3', Anti-sense : $5^{\prime}$ - CAAUAAUCCUUGUGGCAUGCUTT$3^{\prime}$ ), si-NC (sense : $5^{\prime}$ - UUCUCCGAACGUGUCACGUdTdT-3', Anti-sense : $5^{\prime}$ - ACGUGACACGUUCGGAGAAdTdT- $3^{\prime}$ ). HepG2 cells were seeded into 12 -well plates, and transfection of siRNA was conducted by using Lipofectamine ${ }^{\mathrm{TM}} 2000$ transfection reagent (Invitrogen, Shanghai, China) according to the manufacturer's instruction.

\section{Cell Counting Kit-8 Assay}

CCK-8 assay was used to evaluate cell proliferation. Briefly, HepG2 cells were seeded at a density of 4,000 cells per well in 96-well plates and incubated for 1, 2, 3, 4, or 5 days respectively. $10 \mu \mathrm{l}$ CCK-8 (Dojindo Molecular Technologies, Japan) was added to each well, incubated for $4 \mathrm{~h}$, and mixed gently on an orbital shaker for 2 min before the absorbance value (OD) of each well was measured at $450 \mathrm{~nm}$. Experiments were carried out in triplicate.

\section{Apoptosis Assay}

$1^{\star} 10^{5}$ transfected cells were seeded on $6 \mathrm{~cm}$-diameter plates with RPMI-1640 medium containing 10\% FBS for $48 \mathrm{~h}$. Then the cells were harvested, washed twice with ice-cold phosphate-buffered saline (PBS), and re-suspended with binding buffer at a concentration of $1 * 10^{6}$ cells $/ \mathrm{ml}$. The cells were then labeled by using an annexin V-FITC/PI staining kit (eBioscience, United States) according to manufacturer's instructions. The DNA content of labeled cells was analyzed with fluorescence activated cell sorting (FACS) cytometry (Millipore, United States). Experiments were performed in triplicate.

\section{Cell Invasion Assay}

$1 * 10^{5}$ transfected cells were seeded in $500 \mu \mathrm{l}$ RPMI-1640 medium on the matrigel in the upper chamber of the 
Corning $^{\circledR}$ BioCoat $^{\mathrm{TM}}$ Matrigel $^{\circledR}$ Invasion Chambers (8 mm pore size; Corning, United States), $750 \mu \mathrm{l}$ RPMI-1640 medium containing $30 \%$ FBS was added in the bottom chamber. The cells were incubated for $24 \mathrm{~h}$ at $37^{\circ} \mathrm{C}$ with $5 \% \mathrm{CO}_{2}$ and then were fixed in $4 \%$ paraformaldehyde and stained with crystal violet solution (Sigma, United States). The cells on the bottom of the membrane were visualized under a microscope and quantified by counting the number of cells in three randomly chosen fields at 100 -fold magnification. Experiments were performed in triplicate.

\section{IHC Staining}

IHC staining of BAG2 on $8 \mu \mathrm{m}$ paraffin sections was performed according to a standard 3,3'-Diaminobenzidine (DAB) staining protocol (Abclone, China) with primary antibody against BAG2 (Abclone, ab115205, 1:200). Two independent pathologists assessed all IHC samples. The extent of cell staining ( $0-10 \%$ positive cells for $0 ; 11-50 \%$ positive cells for $2 ; 51-80 \%$ positive cells for 3 ; $>80 \%$ positive cells for 4 ) and the staining intensity (no staining for 0 ; slight staining for 1 ; moderate staining for 2 ; strong staining for 3 ) were scored separately and then added to reflect the expression of BAG2 [14].

\section{Western Blot}

Western blotting analysis was conducted as reported in our previous studies [13]. Briefly, transfected cells were cultured for $24 \mathrm{~h}$ before total protein was extracted by using RIPA buffer (Beyotime, China) containing proteinase inhibitor cocktail (Roche, Switzerland). The BCA Protein Assay Kit (Beyotime, China) was used to measure the protein concentration, and approximately $30 \mu \mathrm{g}$ of total protein was separated by $10 \%$ SDS-PAGE and transferred onto PVDF membranes (Millipore, United States). The membranes were incubated in $5 \%$ skim milk for $1 \mathrm{~h}$ at room temperature and then overnight at $4^{\circ} \mathrm{C}$ with primary antibodies. After the membranes were washed three times, they were incubated with HRP-conjugated secondary antibody. The primary antibodies were rabbit polyclonal anti-BAG2 (Abclonal, China) and rabbit polyclonal anti-GAPDH (Abcam, United States). The secondary antibody was HRP-conjugated anti-rabbit (Sigma, United States).

\section{Bioinformatics Analysis and Statistical Analysis}

Normalized gene expression data (HTSeq-FPKM), phenotype data, survival information, and miRNA expression quantification data of liver hepatocellular carcinoma (LIHC) were downloaded from TCGA through GDC Xena Hub (https://xena.ucsc.edu/public) on March 03, 2020. Progression free survival (PFS) and disease specific survival (DSS) data were downloaded from Kaplan Meier-plotter (http://www.kmplot.com), with the gene expression data of BAG2. The Oncomine database (https://www.oncomine.org/ resource/login.html) was used to validate the expression of BAG2 in HCC, with default thresholds: $p$-value $<1 \mathrm{E}-4$, fold change $>2$ and the gene ranks in the top 10\%. GSE64041 (60 pairs of tumor and tumor-adjacent normal samples), GSE25097 (6 liver samples from healthy people, 40 cirrhosis samples, 243 tumor-adjacent normal samples, and 268 tumor samples) and GSE121248 (37 tumor-adjacent normal samples and 70 tumor samples) datasets were downloaded from the Gene Expression Omnibus (GEO) database through R (version 3.6.2), by the GEOquery package. All the data in this part were processed, analyzed and plotted by $\mathrm{R}$ (version 3.6.2), and the following packages were used: BiocManager, clusterProfiler, dplyr, ggplot2, ggpubr, limma, org. Hs.eg.db, plyr, survival, survminer, and tidyverse.

The co-expressed genes of BAG2 were constructed into protein-protein interaction (PPI) networks in the STRING database (http://string-db.org). Cytoscape (version 3.7.2) was then used for the visualization of these networks. The hub genes were then identified as the top 10 nodes based on the score generated by the cytoHubba plug-in in Cytoscape (ranked by degree). Gene ontology (GO) and Kyoto Encyclopedia of Genes and Genomes (KEGG) enrichment analysis were conducted by the clusterProfiler package in $\mathrm{R}$ (version 3.6.2) [15].

Overall survival (OS), PFS, and DSS of high- and low-BAG2 subgroups of HCC patients were compared using the KaplanMeier method with the log-rank test. Cox regression analysis were conducted with SPSS (version 21), to evaluate prognostic factors. The cut-off value of BAG2 was determined by X-tile (version 3.6.1) [16]. Levene's test was used for homogeneity of variance test. When the variance was assumed even, unpaired $t$ test was used to compare the data from two groups and onefactor ANOVA test for multiple groups without post-hoc analysis. Wilcoxon signed rank test was used for the comparison between two groups when the variance was assumed uneven. Correlation analysis between the gene expression of BAG2 and that of the rest genes was conducted by $\mathrm{R}$ software (version 3.6.2) with spearman method. The data from in vitro experiments were analyzed and plotted by Graphpad Prism 8 (GraphPad Software, La Jolla, CA). All statistical tests were two tailed and $p$ value $<0.05$ was considered significant.

\section{RESULTS}

\section{BAG2 Was Up-Regulated in HCC}

To delineate the function of BAG2 in HCC, we first examined its expression pattern in the disease. By analyzing the data from the TCGA_LIHC data set, we found that the transcriptional level of BAG2 was considerably increased in tumor tissues when compared with tumor-adjacent normal tissues (Figure 1A, $p<0.001$ ). Such an expression pattern was supported by three independent GEO data sets, namely GSE64041, GSE25097 and GSE121248 (Figures 1B-D, $p<0.0001)$. We further validated that BAG2 was significantly up-regulated in HCC by comparing its expression between HCC samples and normal samples across five independent cohorts in the Oncomine database 

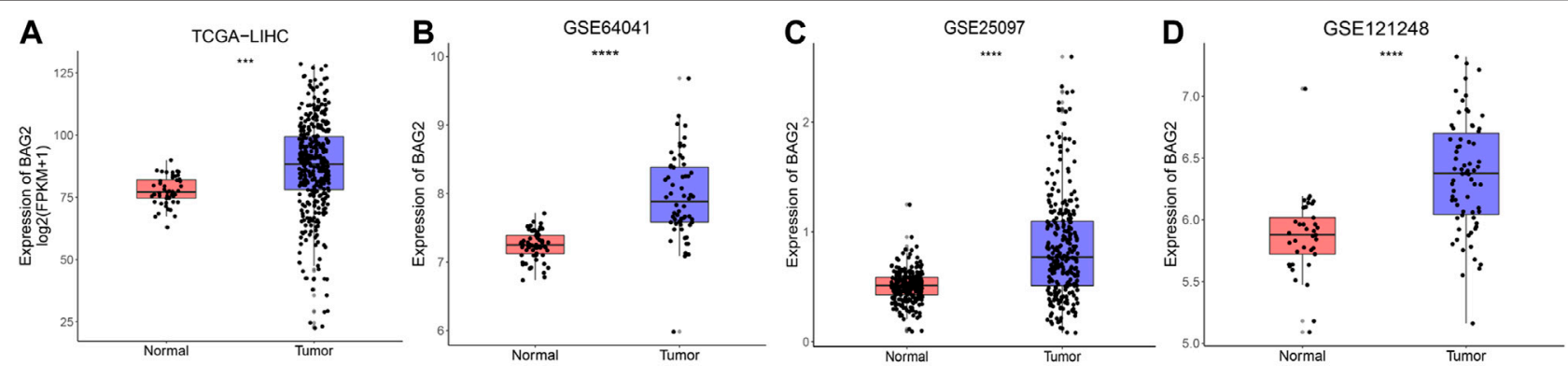

E
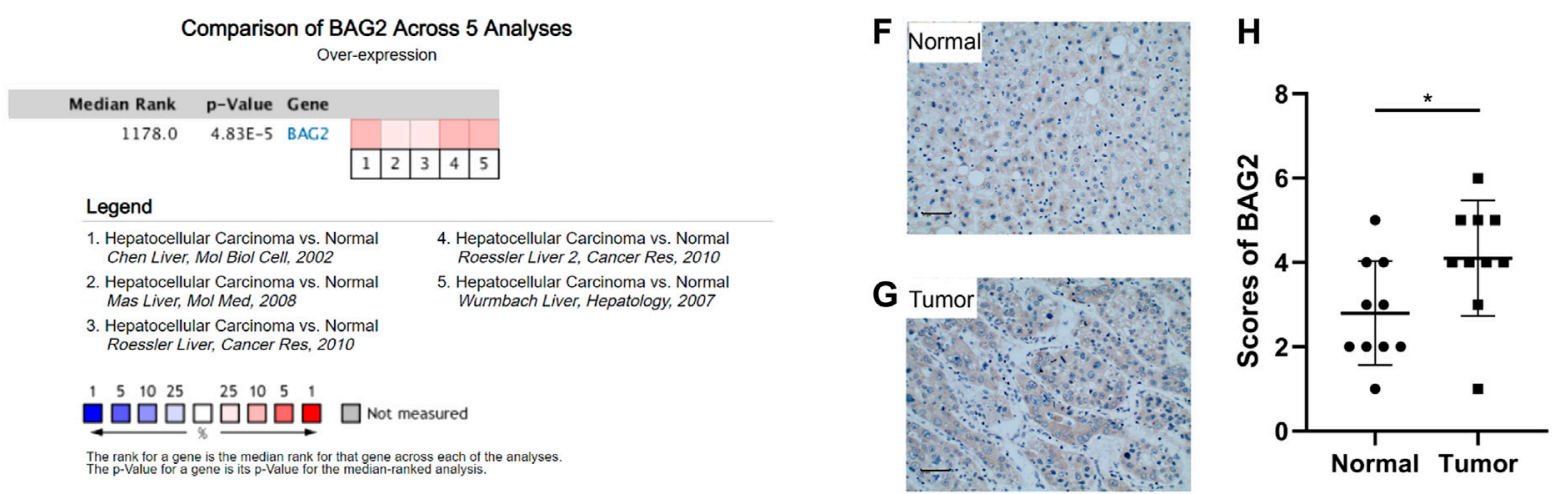

FIGURE 1 | BAG2 was up-regulated in HCC. (A) The gene expression of BAG2 was significantly up-regulated in tumor samples ( $n=374$ ) compared to that in normal livers ( $n=50$ ), based on bioinformatics analysis of TCGA-LIHC dataset. (B-D) Three independent GEO data sets (GSE64041, GSE25097, and GSE121248) showed that BAG2 was overexpressed in HCC tissues when compared with normal tissues. (E) Data from the Oncomine database validated that BAG2 was significantly up-regulated in HCC tissues. (F-H) Quantification of expression level of BAG2 in hepatocellular carcinoma $(n=10)$ and tumor-adjacent tissue $(n=10)$. $p<0.05$ was statistically significant, ns: no significant, ${ }^{\star} p<0.05,{ }^{\star \star} p<0.01,{ }^{\star \star \star} p<0.001,{ }^{\star \star \star \star} p<0.0001$

(Figure 1E). In addition, we conducted IHC staining of BAG2 on 10 HCC tissues and 10 tumor-adjacent normal tissues and scored the staining based on the intensity and extent [14]. The results confirmed that the expression of BAG2 was significantly elevated in tumor tissues when compared with normal tissues (Figures $\mathbf{1 F}-\mathbf{H}, p<0.05$ ).

\section{BAG2 Was Associated With Worse Clinicopathological Features of HCC}

We then examined the relationship between clinicopathological features of HCC patients and the gene expression of BAG2 by manipulating data from the TCGA_LIHC data set. The clinical characteristics of HCC patients were summarized in Supplementary Table S3. A total of 368 cases had gene expression data, survival data and phenotype data. Unavailable or unknown clinical features of the 368 patients were regarded as missing values. Briefly, $44.8 \%$ of HCC patients were younger than 60 years old, and more patients were male than female (67.7 vs. $32.3 \%$ ). Most patients were at relatively good status, because only a small fraction of patients were at T4 stage (3.5\%), with lymph node metastasis (N1, 1.1\%), with distant metastasis (M1, $0.8 \%)$, diagnosed as stage IV (1.1\%), or with bad liver function (Child-Pugh classification C, 0.3\%). Besides, 56.3\% of patients had no vascular invasion, $20.4 \%$ had no fibrosis, and $32.1 \%$ had no adjacent hepatic tissue inflammation. With respect to cancer status, $33.7 \%$ of HCC patients had tumor while $44.0 \%$ of them were tumor free. As shown in Figures 2A-I, high level of BAG2 was significantly correlated with advanced T stage $(p<0.01)$ and disease stage $(p<0.05)$. Due to the small number of patients with lymph node or distant metastasis, no correlation was found between $\mathrm{N}$ or $\mathrm{M}$ stage and BAG2 expression. In addition, patients with poor cancer status (with tumor, $p<0.05$ ) or with vascular invasion $(p<$ 0.05 ) was associated with higher expression of BAG2. No correlation was found between the expression of BAG2 and level of AFP, fibrosis or adjacent hepatic tissue inflammation.

As shown in Figures 2J-L, HCC patients with high expression of BAG2 had significantly shorter overall survival (OS, $p=0.00012$ ), progression free survival (PFS, $p=0.0029$ ), and disease specific survival (DSS, $p=0.007)$. A univariate analysis revealed that low level of BAG2 [hazard ratio (HR): 0.508; 95\% confidence interval (CI): $0.357-0.722 ; p<0.001$ ], good cancer status (HR: 0.372, CI: $0.234-0.590 ; p<0.001$ ), early T stage (HR: 0.479, CI: $0.334-0.685$; $p<0.001$ ), without lymph node metastasis (HR: 0.676, CI: $0.469-0.973 ; p=0.035$ ), and without distant metastasis (HR: 0.613 , CI: $0.426-0.883 ; p=0.009)$ were all associated with longer OS (Table 1a). We further put these factors into a multivariate Cox regression analysis and found that low BAG2 expression was still an independent factor that correlated to good prognosis (HR: 0.421, CI: 0.247-0.719; $p=0.002$ ) (Table 1b). 


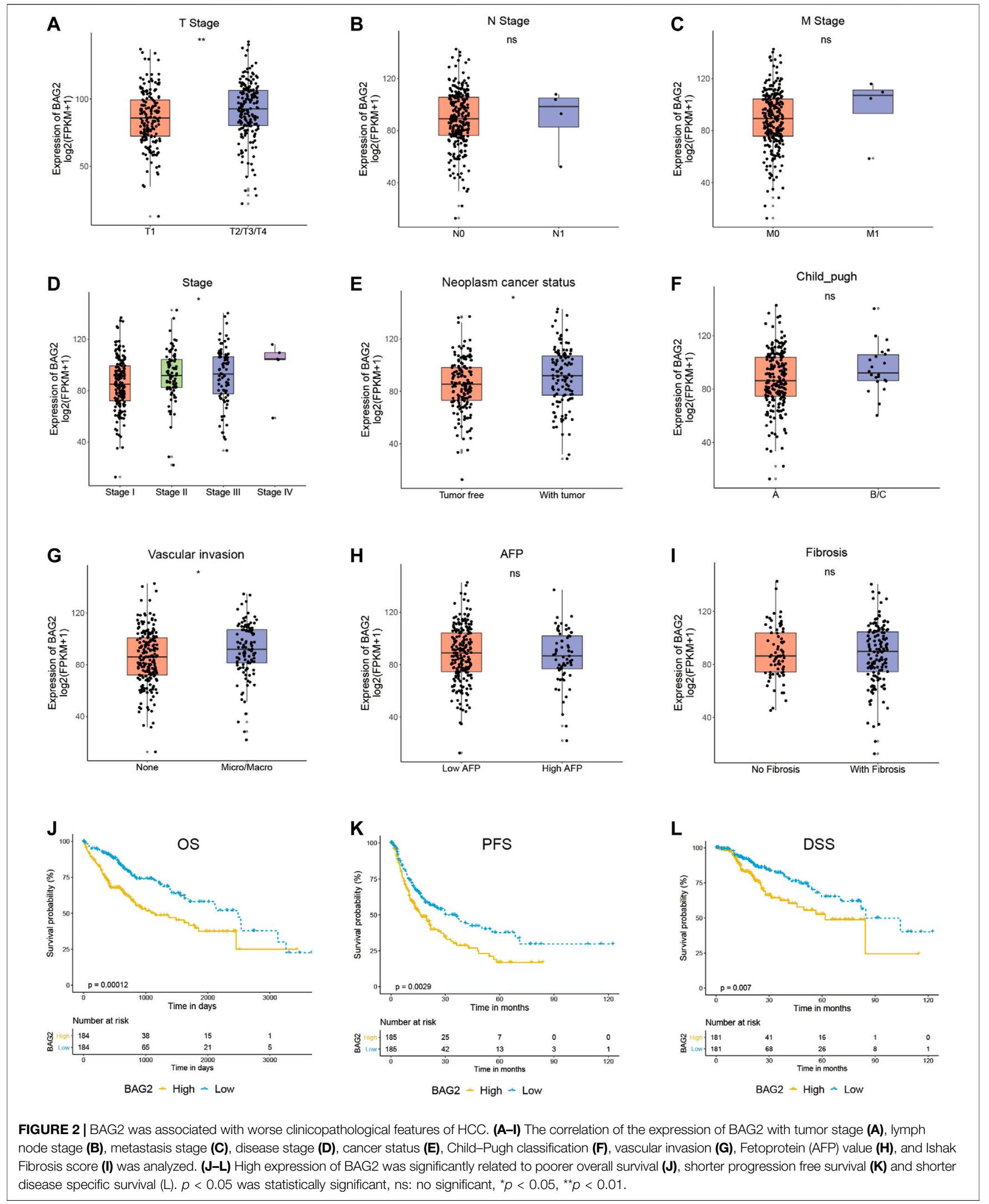


TABLE 1 | a. Correlation between clinicopathological characteristics and overall survival and of HCC patients by using Cox regression. b. Multivariate survival analysis after variable selection.

\begin{tabular}{|c|c|c|}
\hline Clinicopathologic variable & HR (95\% Cl) & $p$-value \\
\hline \multicolumn{3}{|l|}{ a } \\
\hline BAG2 (low vs. high) & $0.508(0.357-0.722)$ & $<0.001$ \\
\hline Inflamation(None vs. Mild/Severe) & $0.869(0.535-1.406)$ & 0.564 \\
\hline Person neoplasm cancer status (tumor free vs. With tumor) & $0.372(0.234-0.590)$ & $<0.001$ \\
\hline Age (<60 vs. $\geq 60)$ & $0.857(0.603-1.220)$ & 0.393 \\
\hline Race (non-white vs. White) & $0.802(0.559-1.149)$ & 0.229 \\
\hline Gender (male vs. female) & $0.801(0.563-1.141)$ & 0.219 \\
\hline Vascular tumor (None vs. Micro/Macro) & $0.758(0.501-1.147)$ & 0.19 \\
\hline Stage (I vs. II-IV) & $0.491(0.337-0.716)$ & $<0.001$ \\
\hline T (T1 vs. T2/T3/T4) & $0.479(0.334-0.685)$ & $<0.001$ \\
\hline N (NO vs. N1/NX) & $0.676(0.469-0.973)$ & 0.035 \\
\hline $\mathrm{M}(\mathrm{M} 0$ vs. M1/MX) & $0.613(0.426-0.883)$ & 0.009 \\
\hline Fibrosis score (0 vs. 1-6) & $1.350(0.818-2.229)$ & 0.24 \\
\hline AFP (<400 vs. $\geq 400)$ & $0.944(0.578-1.542)$ & 0.818 \\
\hline Child-pugh (A vs. B/C) & $0.609(0.300-1.234)$ & 0.168 \\
\hline \multicolumn{3}{|l|}{$\mathrm{b}$} \\
\hline BAG2 (low vs. high) & $0.421(0.247-0.719)$ & 0.002 \\
\hline Person neoplasm cancer status (tumor free vs. With tumor) & $0.566(0.337-0.951)$ & 0.032 \\
\hline Stage (I vs. II-IV) & $0.382(0.043-3.384)$ & 0.388 \\
\hline T (T1 vs. T2/T3/T4) & 1.023 (0.122-8.547) & 0.983 \\
\hline N (N0 vs. N1/NX) & $0.889(0.419-1.884)$ & 0.758 \\
\hline M (M0 vs. M1/MX) & $1.001(0.465-2.156)$ & 0.999 \\
\hline
\end{tabular}

\section{Silencing BAG2 Facilitated Apoptosis, and Repressed Proliferation and Invasion of HCC Cells}

To investigate how BAG2 affected biological activities of HCC, we transfected HepG2 cells with three si-BAG2s or si-NC. All the three si-BAG2s dramatically down-regulated the expression of BAG2 on the mRNA and protein level, and si-BAG2-2 had the strongest inhibition (Figure 3A). We then used si-BAG2-2 for further experiments. As shown in Figure 3B, silencing the expression of BAG2 in HCC cells significantly impaired cell proliferation on day $4(p<0.01)$ and day $5(p<0.01)$. Besides, HCC cells with down-regulated expression of BAG2 showed a significantly increased percentage of apoptotic cells (Figure 3C, $p<0.01$ ). Because Lisha Sun et al. reported that BAG2 promoted metastasis of gastric cancer [7], we further conducted invasion assay and the result suggested that BAG2 knockdown dramatically decreased the amount of cells invaded though the matrigel (Figure 3D).

\section{BAG2 Might Regulate Ribosome Biogenesis}

The above analysis and experiments indicated that BAG2 was upregulated in HCC and might favor the progression of the disease; we then intended to understand the underlying regulatory mechanisms. Correlation analysis was conducted independently between the expression of BAG2 and that of other genes in tumor samples of the TCGA_LIHC and GSE64041 data sets. 10,918 genes and 3,837 genes were identified as co-expressed genes in the TCGA_LIHC and GSE64041 data sets respectively ( $p$ value cutoff $=0.05$ ). Based on the correlation coefficient, the top 500 co-expressed genes from the two data sets were intersected and 155 genes were found to be the common co-expressed genes for BAG2 (Figure 4A; Supplementary Table S4). These co-expressed genes were then undergoing GO and KEGG analysis. The significantly affected biological processes (BP) were "RNA biogenesis and processing like ribosome biogenesis," "ribosome RNA (rRNA) metabolic process," "non-coding RNA (ncRNA) processing," "rRNA processing," "maturation of SSU-rRNA from tricistronic rRNA transcript (SSU-rRNA, 5.8S rRNA, LSU-rRNA)," and "positive regulation of telomerase RNA localization to Cajal body" (Figure 4B). The significantly enriched molecular functions (MF) of these co-expressed genes were "catalytic activity," "unfolded protein binding," "RNA polymerase activity," and "aminoacyl-tRNA ligase activity" (Figure 4B). According to the KEGG analysis, "RNA transport," "RNA polymerase," "aminoacyl-tRNA biosynthesis," "Alanine, aspartate and glutamate metabolism," and "ribosome biogenesis in eukaryotes" were significantly enriched terms (Figure 4C; Table 2).

\section{PPI Network Construction and Analysis of Hub Genes}

We then uploaded BAG2 and its 155 co-expressed genes into the STRING database and constructed a BAG2-contained PPI network, which was visualized by the Cytoscape software (Figure 5A). By using the cytoHubba plug-in, the 10 hub genes (ranked by degree) were identified to be WD repeat domain 12 (WDR12), bystin like (BYSL), DDB1 and CUL4 associated factor 13 (DCAF13), WD repeat domain 3 (WDR3), chaperonin containing TCP1 subunit 2 (CCT2), ribosome biogenesis regulator 1 homolog (RRS1), nucleolar pre-RRNA processing protein NIP7 (NIP7), RNA polymerase 

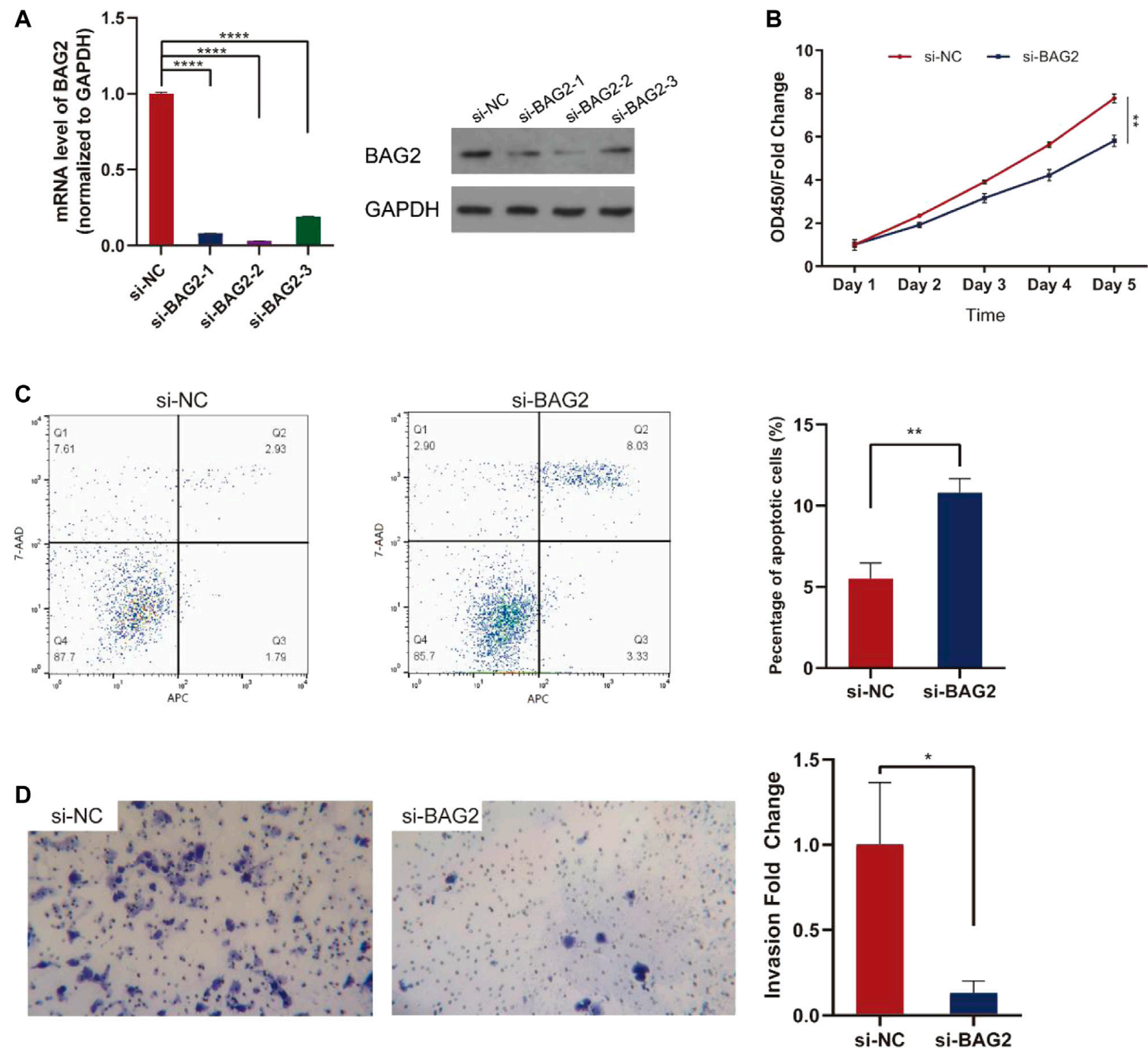

FIGURE 3 | BAG2 suppressed apoptosis, favored proliferation and invasion of HCC. (A) Three si-BAG2s dramatically suppressed the expression of BAG2 in HepG2 cells (B) CCK-8 assay indicated that HepG2 cells transfected with si-BAG2 had decreased cell proliferation (C) Down-regulation of BAG2 in HepG2 cells led to increased percentage of apoptotic cells (D) Down-regulation of BAG2 in HepG2 cells impaired the invasion capability of tumor cells. All values shown were mean \pm SD. $p<0.05$ was statistically significant, ${ }^{\star} p<0.05,{ }^{* \star} p<0.01,{ }^{\star \star *} p<0.001,{ }^{\star * \star *} p<0.0001$.

I and III subunit C (POLR1C), nucleolar protein interacting with the FHA domain of MKI67 (NIFK), chaperonin containing TCP1 subunit 7 (CCT7) (Figure 5A, yellow blocks; Supplementary Table S4). The biological process analysis of these hub genes was conducted by using the BINGO plug-in the Cytoscape software. "Ribosome biogenesis," "ribosomal large subunit biogenesis," "ribonucleoprotein complex biogenesis," "rRNA processing," "ncRNA processing," "rRNA metabolic process," "RNA processing," "arginyl-tRNA aminoacylation" and "ncRNA metabolic process" were significantly altered, consistent with the GO and KEGG enrichment analysis of the co-expressed genes of BAG2 (yellow modules, Figure 5B). Except WDR3, all the rest nine hub genes were significantly up-regulated in tumors when compared to tumor-adjacent normal tissues in both the TCGA_LIHC and GE64041 data sets (Figures 5C,D). We then analyzed the prognostic significance of the nine hub genes in the TCGA_LIHC data set, and the result suggested that WDR12, BYSL, DCAF13, CCT2, RRS1, NIP7, NIFK, and CCT7 at high expression levels were all associated with significantly unfavorable prognosis in HCC (Figures 6A-H), while no significant correlation was observed between the expression of POLR1C and OS of HCC patients (Figure 6I). In addition, previous studies revealed that most of these hub genes were required or inducible for ribosome biogenesis [17-23]. Further, 
A

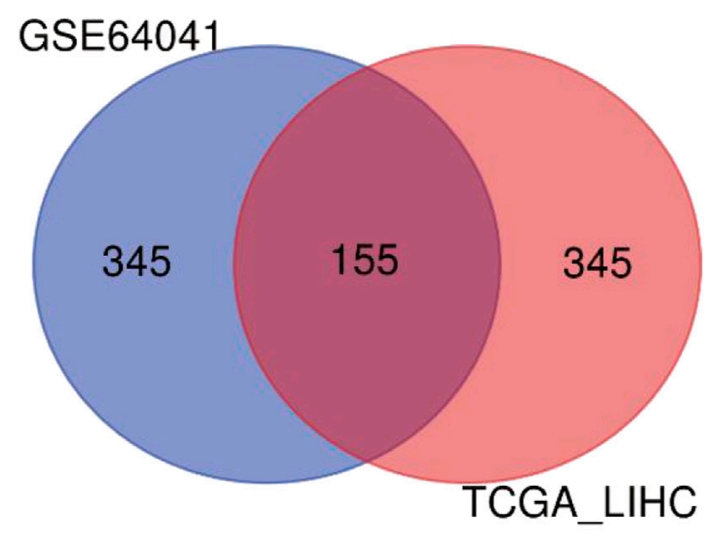

B

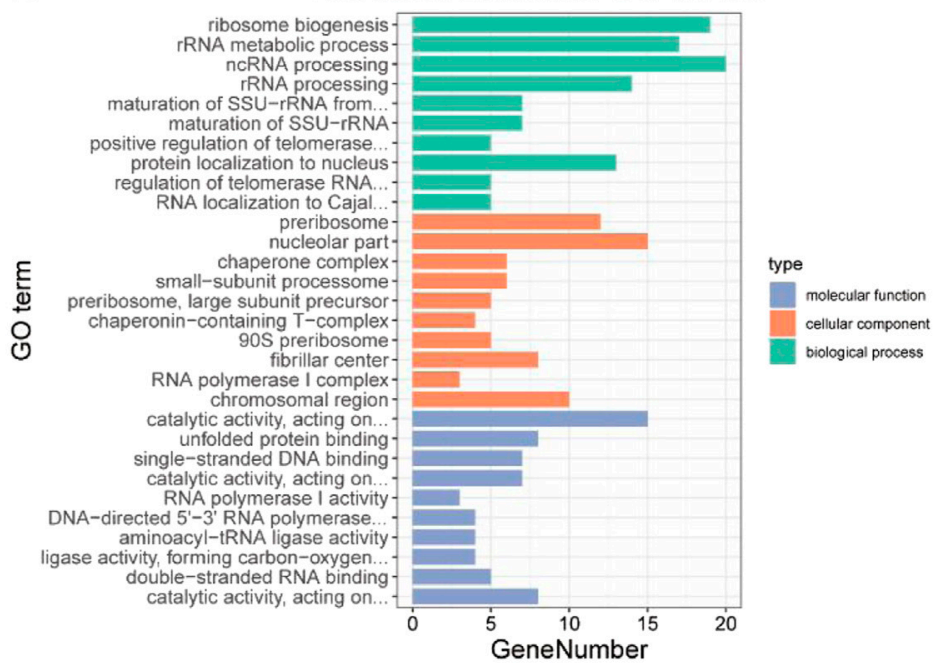

C

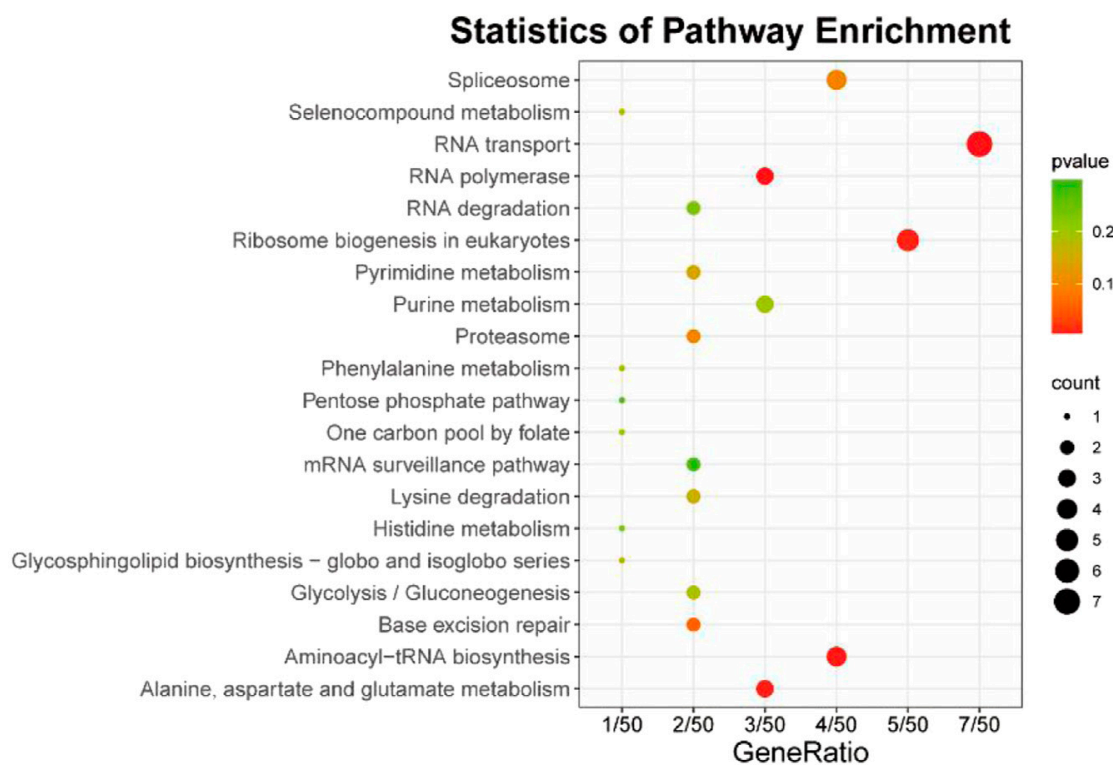

FIGURE 4 | Co-expression analysis and subsequent GO and KEGG enrichment analysis of BAG2. (A) Co-expression analysis of BAG2 in TCGA_LIHC and GSE64041 data sets. (B) GO enrichment analysis of the common co-expressed genes of BAG2. (C) KEGG enrichment analysis of the common co-expressed genes of BAG2.

TABLE 2 | KEGG analysis of the co-expressed genes of BAG2 in HCC.

ID

Description

$p$ value

hsa03013

RNA transport

0.004534547

hsa03020

RNA polymerase

Aminoacyl-tRNA biosynthesis

hsa00250

Alanine, aspartate and glutamate metabolism

hsa03008

Ribosome biogenesis in eukaryotes

0.005399698

0.007045845

0.008232431

0.009074458

we detected the transcriptional level of the 10 hub genes in HepG2 cells with BAG2 silencing, and found that all of them showed a significant down-regulation after BAG2 knockdown, suggesting
BAG2 might affect ribosome biogenesis through these genes (Supplementary Figure S1).

\section{DISCUSSION}

Identifying oncogenes and their related regulatory mechanisms in tumors is of great help in developing new potential therapies. For instance, cetuximab targeting epidermal growth factor receptor (EGFR), bevacizumab against vascular endothelial growth factor receptor (VEGFR), and dabrafenib inhibiting BRAF V600E mutation has become cornerstone of anti-tumor treatments and considerably improve the prognosis of advanced cancer patients [24-26]. 


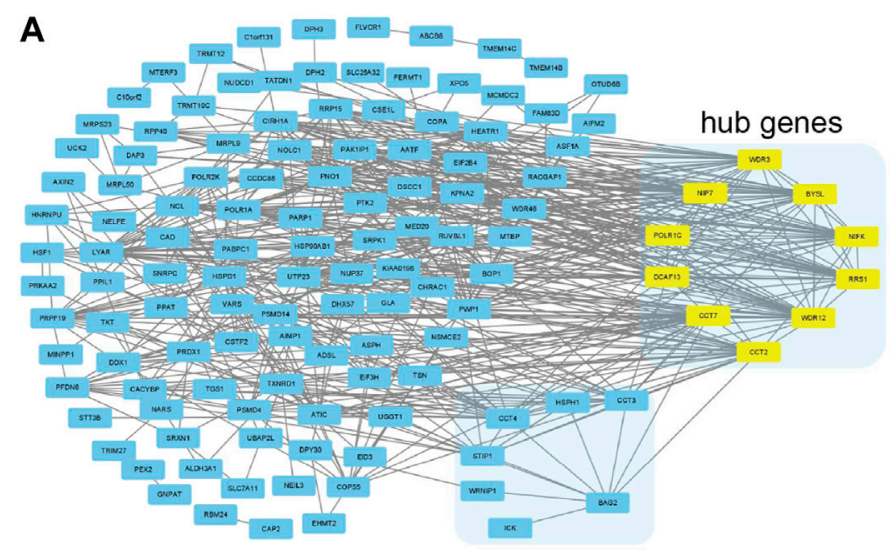

B

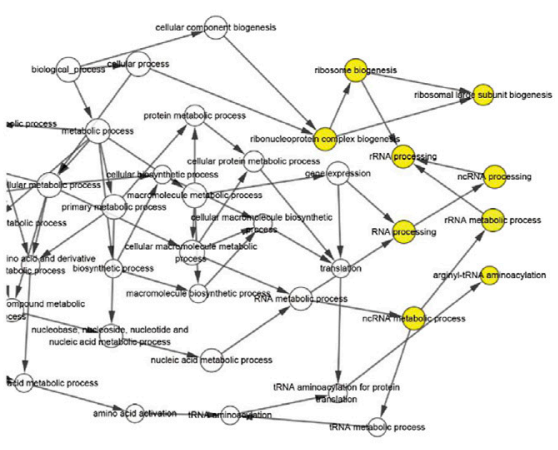

C

TCGA_LIHC

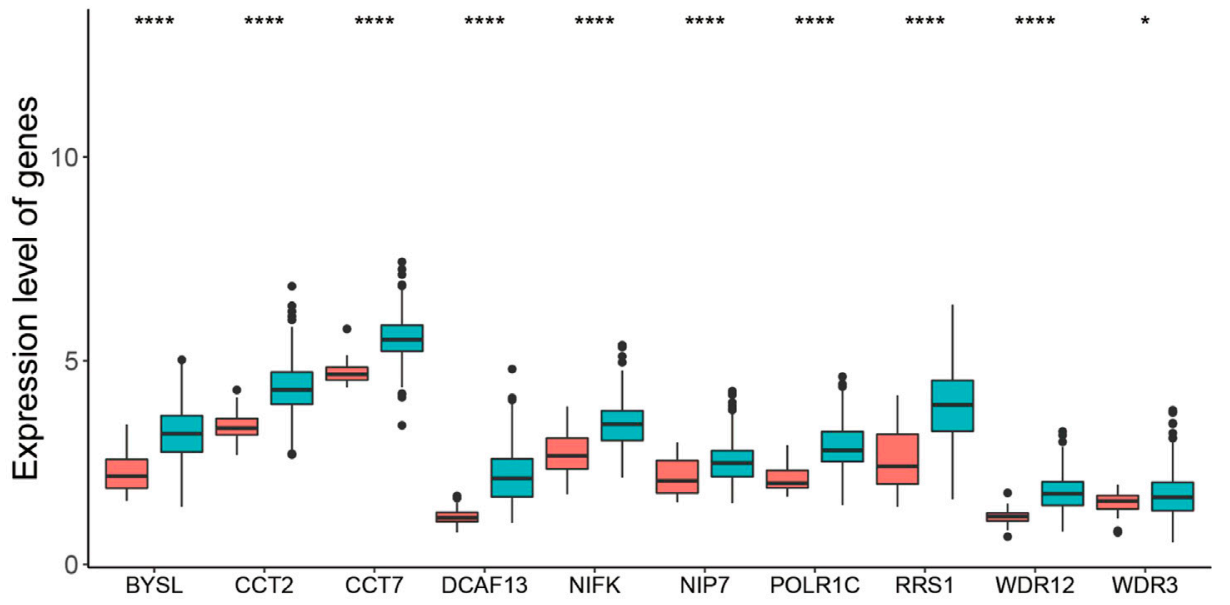

D

GSE64041

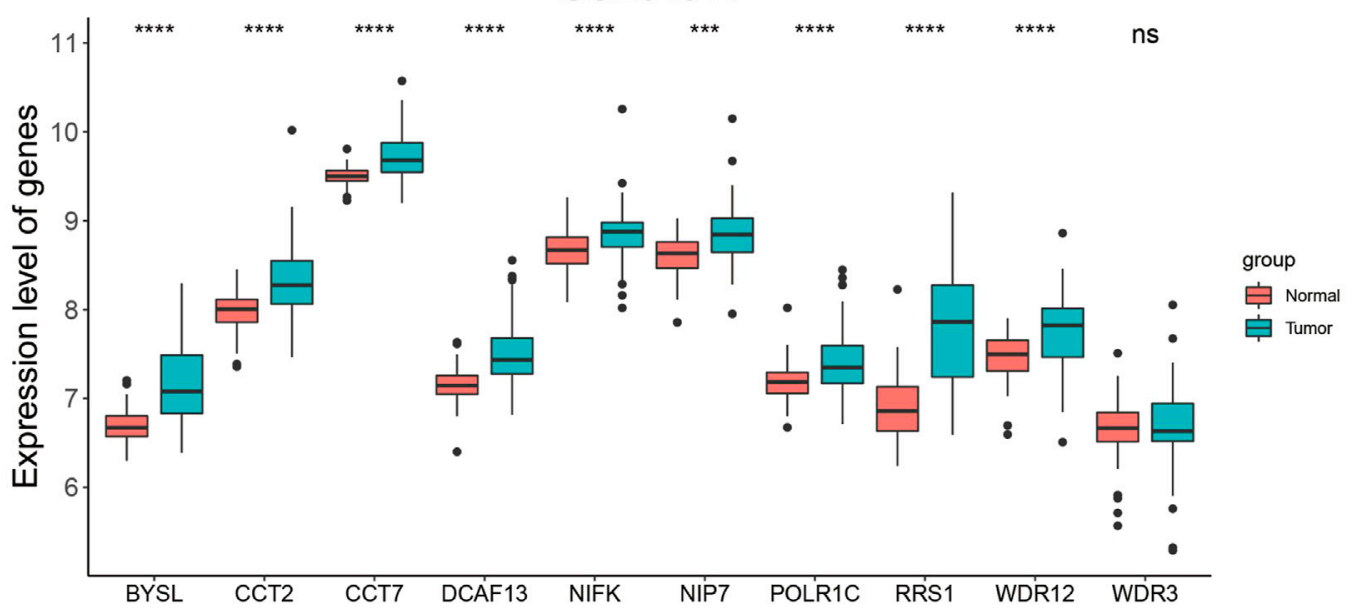

FIGURE 5 |PPI network construction and analysis of hub genes. (A) PPI network construction of the co-expressed genes of BAG2 and identification of hub genes (yellow block). (B) Biological process analysis of hub genes by using the BINGO plug-in in the Cytoscape software. (C,D) Expression analysis of the 10 hub genes in tumor and non-tumor samples in TCGA_LIHC (C) and GSE64041 (D) data sets. $p<0.05$ was statistically significant, ${ }^{*} p<0.05,{ }^{* \star} p<0.01,{ }^{\star \star \star} p<0.001,{ }^{\star \star \star \star} p<0.0001$. 


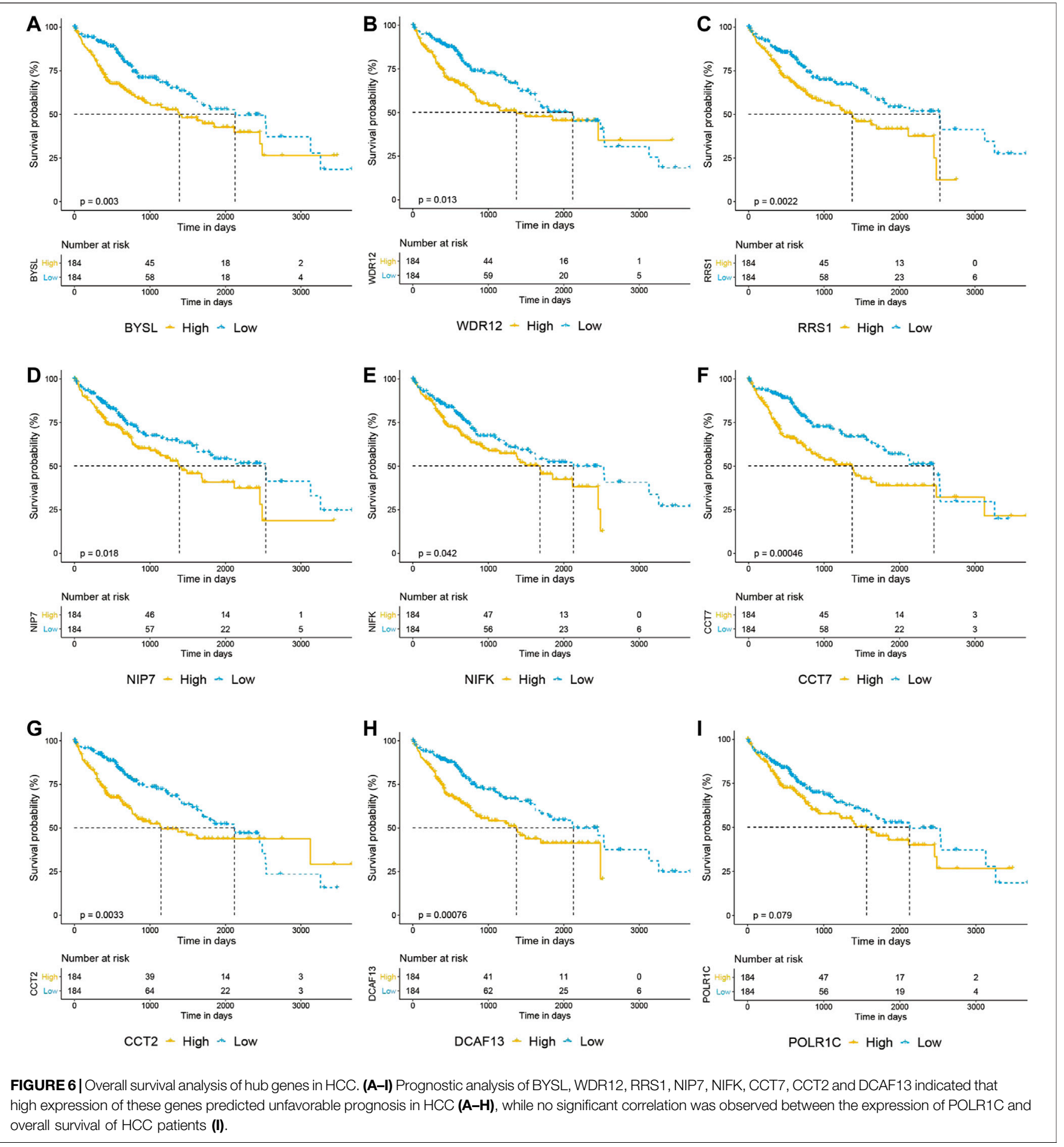

The BAG family, which is evolutionarily conserved, might serve as a new potential therapeutic target in cancer treatment since studies show that members of this family are related to chemo-resistance, invasion and apoptosis of tumors [27-31]. For instance, Tugba Kizilboga et al. reported that BAG1 favored survival of breast cancer cells by phosphorylating the proapoptotic Bad protein through activation of the Akt and Raf kinase pathways [30]. Anja Bruchmann et al. found that BAG5 was over-expressed in prostate cancer and played a role in preventing stress-induced cell death [31]. Jiling Lv et al. showed that down-regulation of BAG1 could sensitize nonsmall cell lung cancer (NSCLC) cells to chemotherapy like cisplatin [28].

Some studies also investigated the role of BAG family in HCC. Wenkai Ni et al. reported that BAG1 was over-expressed in the nucleus of HCC cells and high expression of the gene increased 
resistance of HCC cells to doxorubicin [32]. The role of BAG3 in HCC is somewhat controversial, since Dehui Kong et al. showed that high expression of BAG3 suppressed cell proliferation while Heng Xiao et al. reported that BAG3 elevation favored invasiveness and angiogenesis in $\operatorname{HCC}[33,34]$. In this study, we for the first time, to our knowledge, investigated the role of BAG2 in HCC. Bioinformatics analysis of the data from TCGA and GEO data sets, and IHC staining of BAG2 on collected tumor tissues and tumor-adjacent normal tissues all showed that BAG2 was significantly up-regulated in HCC when compared with normal liver samples. A similar expression pattern of BAG1 and BAG3 has been reported in HCC, indicating that the BAG family might actively involve in the development of the disease $[32,33]$. Elevated expression of BAG2 was further found to be associated with worse clinicopathological features. In vitro experiments showed that BAG2 might play a role in many biological behaviors of HCC, like favoring cell proliferation, repressing apoptosis and favoring tumor invasion (Figures 3A-E). With these pro-tumor effects in HCC, BAG2 at higher levels was found to be related to shorter survival of HCC patients and could serve as an independent prognostic marker (Figures 2J-L; Table 2). Other studies also revealed that BAG2 supported proliferation and/or metastasis of tumors like breast cancer and oral cancer $[7,9,10]$. Taken together, BAG2 might act as an oncogene across a set of tumors.

To understand the underlying mechanism of BAG2 in favoring proliferation and invasion of HCC, we conducted co-expression analysis in the TCGA_LIHC and GSE64041 data sets (Figure 4A). GO and KEGG enrichment analysis showed that BAG2 might regulate ribosome biogenesis of HCC (Figures 4B,C). Indeed, previous studies found that BYSL, NIP7, DCAF13, WDR12, NIFK, and RRS1 regulated ribosome biogenesis [17-23]. These genes were found to be hub genes of BAG2, and they were down-regulated in HCC cells after silencing of BAG2 (Supplementary Figure S1). Ribosome biogenesis is an old topic, but gains accumulating attention in cancers in recent years $[2,35]$. Researchers find that human pathological conditions characterized by an upregulated ribosome biogenesis are at an increased risk of cancer onset, and ribosome biogenesis not only supports cell proliferation but facilitates tumor invasion [2, 3, 36]. P53 plays an important role in balancing ribosome biogenesis rate and cell cycle progression [36]. Studies showed that p53 negatively controlled ribosome biogenesis, by inhibiting transcription of both nuclear RNA polymerases I and III, two key determinants for the ribosome production [37, 38]. However, cancer cells harboring p53 mutation might lose its negative control on ribosome biogenesis [36]. Since BAG2 contributes to the accumulation of mutant $\mathrm{p} 53$, which could interact with c-Myc and enhance c-Myc-dependent rDNA transcription for ribosome biogenesis, mutant p53 might be a downstream mediator of BAG2 in regulating the biogenesis $[8,39]$. It is worthy to point out that mutant p53 occurs frequently in HCC and related to poor differentiation and vascular invasion [40, 41]. Further studies are required to determine whether BAG2 plays a predominant role in the accumulation of mutant $\mathrm{p} 53$ protein. In addition, PI3K/Akt/
mTOR and MAPK signaling pathways might be other potential downstream mediators of BAG2 in regulating ribosome biogenesis, as these pathways were reported to regulate ribosome biogenesis and could be modulated by other members of the BAG family [27, 29, 30, 42].

Besides, it is possible that BAG2 regulates apoptosis, proliferation and invasion via mechanisms other than ribosome biogenesis regulation, since BAG proteins could functionally interact with a variety of binding partners including Hsp70 [5, 43]. Indeed, the BAG2-Hsp70 complex plays an essential role in the protein homeostasis [43]. Studies found that Hsp70 suppressed apoptosis through multiple pathways, like preventing Bax translocation, preventing oligomerization of apoptotic protease activating factor 1 (APAF1), neutralizing the apoptosis-inducing factor (AIF) and inhibiting the activation of Caspase-3 [44-48]. Moreover, the BAG family is well known for its capability in preventing cell death through interaction with $\mathrm{Bcl}-2$, which plays a prominent role in suppressing apoptosis and enhancing cell survival in response to diverse apoptotic stimuli [5, 49]. Thus, BAG2 might inhibit cell apoptosis in HCC via enhancing the anti-apoptotic function of Bcl-2 [50]. Lisha Sun et al. reported that BAG2 could bind to ERK1/2 in gastric cancer, and promote proliferation and metastasis of the disease [7]. Numerous studies showed that ERK1/2 promoted progression of HCC, thus the signaling might be an important downstream pathway of BAG2 in the disease [51-53]. In addition, BAG2 could interact with pro-peptide region of pro-cathepsin B, block the auto-cleavage process and facilitate the secretion of the enzyme, which induces metastasis of TNBC [9]. Since cathepsin B participates in the progression of HCC, it would be of great importance to investigate the BAG2/cathepsin B axis in HCC by future studies $[54,55]$.

\section{CONCLUSION}

In summary, this work showed that BAG2 was up-regulated in HCC and high expression of the gene correlated to unfavorable prognosis in the disease. BAG2 might favor cell proliferation, repress apoptosis and facilitate tumor invasion in HCC, probably through regulation on ribosome biogenesis via genes like WDR12, BYSL, and DCAF13 [17, 19, 20].

\section{DATA AVAILABILITY STATEMENT}

The original contributions presented in the study are included in the article/Supplementary Material, further inquiries can be directed to the corresponding author.

\section{ETHICS STATEMENT}

The studies involving human participants were reviewed and approved by the institutional review board (IRB) of the Third Xiangya Hospital, Central South University. The patients/ participants provided their written informed consent to participate in this study. 


\section{AUTHOR CONTRIBUTIONS}

The study was conceived and designed by ZS. In vitro experiments and collection of data was done by XZ. Data analysis was carried out by XZ, YL and ZS. IHC staining and scoring was conducted by $\mathrm{YL}$ and JT independently. Bioinformatics analysis was conducted by ZS and JZ. Useful discussion and advice were given by JL. ZS drafted the article, and all authors revised the article and approved the final version to be published.

\section{FUNDING}

This work is supported by the Youth Program of National Natural Science Foundation of China (Grant No. 81802940). This work is supported by the New Xiangya Talent Project of the Third Xiangya Hospital of Central South University (Grant No. JY201715).

\section{REFERENCES}

1. Pelletier J, Thomas G, Volarević S. Ribosome biogenesis in cancer: new players and therapeutic avenues. Nat Rev Cancer (2018) 18(1):51-63. doi:10.1038/nrc. 2017.104

2. Penzo M, Montanaro L, Treré D, Derenzini M. The ribosome biogenesis-cancer connection. Cells (2019) 8(1):55. doi:10.3390/cells8010055

3. Prakash V, Carson BB, Feenstra JM, Dass RA, Sekyrova P, Hoshino A, et al. Ribosome biogenesis during cell cycle arrest fuels EMT in development and disease. Nat Commun (2019) 10(1):2110. doi:10.1038/s41467-019-10100-8

4. Khot A, Brajanovski N, Cameron DP, Hein N, Maclachlan KH, Sanij E, et al. First-in-Human RNA polymerase I transcription inhibitor CX-5461 in patients with advanced hematologic cancers: results of a phase I dose-escalation study. Cancer Discov (2019) 9(8):1036-49. doi:10.1158/2159-8290.cd-18-1455

5. Mariotto E, Viola G, Zanon C, Aveic S. A BAG's life: every connection matters in cancer. Pharmacol Ther (2020) 209:107498. doi:10.1016/j.pharmthera.2020. 107498

6. Lee J-H, Takahashi T, Yasuhara N, Inazawa J, Kamada S, Tsujimoto Y. Bis, a Bcl-2-binding protein that synergizes with $\mathrm{Bcl}-2$ in preventing cell death. Oncogene (1999) 18(46):6183-90. doi:10.1038/sj.onc.1203043

7. Sun L, Chen G, Sun A, Wang Z, Huang H, Gao Z, et al. BAG2 promotes proliferation and metastasis of gastric cancer via ERK1/2 signaling and partially regulated by miR186. Front Oncol (2020) 10:31. doi:10.3389/fonc.2020.00031

8. Yue X, Zhao Y, Liu J, Zhang C, Yu H, Wang J, et al. BAG2 promotes tumorigenesis through enhancing mutant p53 protein levels and function. Elife (2015) 4:e08401. doi:10.7554/elife.08401

9. Yang K-M, Bae E, Ahn SG, Pang K, Park Y, Park J, et al. Co-chaperone BAG2 determines the pro-oncogenic role of cathepsin B in triple-negative breast cancer cells. Cel Rep (2017) 21(10):2952-64. doi:10.1016/j.celrep.2017.11.026

10. Liu Y-S, Wei B. Over-expression of $\mathrm{Bcl} 2$-associated athanogene 2 in oral cancer promotes cellular proliferation and is associated with poor prognosis. Arch Oral Biol (2019) 102:164-70. doi:10.1016/j.archoralbio.2019.04.015

11. Wang H-Q, Zhang H-Y, Hao F-J, Meng X, Guan Y, Du Z-X. Induction of BAG2 protein during proteasome inhibitor-induced apoptosis in thyroid carcinoma cells. Br J Pharmacol (2008) 155(5):655-60. doi:10.1038/bjp. 2008.302

12. Song Z, Yu Z, Chen L, Zhou Z, Zou Q, Liu Y. MicroRNA-1181 supports the growth of hepatocellular carcinoma by repressing AXIN1. Biomed Pharmacother (2019) 119:109397. doi:10.1016/j.biopha.2019.109397

13. Song Z, Fusco J, Zimmerman R, Fischbach S, Chen C, Ricks DM, et al. Epidermal growth factor receptor signaling regulates $\beta$ cell proliferation in adult mice. J Biol Chem (2016) 291(43):22630-7. doi:10.1074/jbc.m116. 747840

\section{CONFLICT OF INTEREST}

The authors declare that the research was conducted in the absence of any commercial or financial relationships that could be construed as a potential conflict of interest.

\section{ACKNOWLEDGMENTS}

In addition, we thank Zheng Zhu for providing technical assistance in bioinformatics analysis.

\section{SUPPLEMENTARY MATERIAL}

The Supplementary Material for this article can be found online at: https://www.por-journal.com/articles/10.3389/pore.2021.594649/ full\#supplementary-material.

14. Huang J-Z, Chen M, Chen D, Gao X-C, Zhu S, Huang H, et al. A peptide encoded by a putative IncRNA HOXB-AS3 suppresses colon cancer growth. Mol Cel (2017) 68(1):171-84. doi:10.1016/j.molcel.2017.09.015

15. Yu G, Wang L-G, Han Y, He Q-Y. clusterProfiler: an R package for comparing biological themes among gene clusters. OMICS: A J Integr Biol (2012) 16(5): 284-7. doi:10.1089/omi.2011.0118

16. Camp RL, Dolled-Filhart M, Rimm DL. X-Tile: a new bio-informatics tool for biomarker assessment and outcome-based cut-point optimization. Clin Cancer Res (2004) 10(21):7252-9. doi:10.1158/1078-0432.ccr-04-0713

17. Adachi K, Soeta-Saneyoshi C, Sagara H, Iwakura Y. Crucial role of Bysl in mammalian preimplantation development as an integral factor for $40 \mathrm{~S}$ ribosome biogenesis. $M c b$ (2007) 27(6):2202-14. doi:10.1128/mcb.01908-06

18. Morello LG, Coltri PP, Quaresma AJC., Simabuco FM, Silva TCL, Singh G, et al. The human nucleolar protein FTSJ3 associates with NIP7 and functions in pre-rRNA processing. PLoS One (2011) 6(12):e29174. doi:10.1371/journal. pone.0029174

19. Zhang J, Zhang Y-L, Zhao L-W, Guo J-X, Yu J-L, Ji S-Y, et al. Mammalian nucleolar protein DCAF13 is essential for ovarian follicle maintenance and oocyte growth by mediating rRNA processing. Cell Death Differ (2019) 26(7): 1251-66. doi:10.1038/s41418-018-0203-7

20. Hölzel M, Rohrmoser M, Schlee M, Grimm T, Harasim T, Malamoussi A, et al. Mammalian WDR12 is a novel member of the Pes1-Bop1 complex and is required for ribosome biogenesis and cell proliferation. J Cel Biol (2005) 170(3):367-78. doi:10.1083/jcb.200501141

21. Talkish J, Campbell IW, Sahasranaman A, Jakovljevic J, Woolford JL. Ribosome assembly factors Pwp1 and Nop12 are important for folding of 5.8S rRNA during ribosome biogenesis in Saccharomyces cerevisiae. Mol Cell Biol (2014) 34(10):1863-77. doi:10.1128/mcb.01322-13

22. Pan W-A, Tsai H-Y, Wang S-C, Hsiao M, Wu P-Y, Tsai M-D. The RNA recognition motif of NIFK is required for rRNA maturation during cell cycle progression. RNA Biol (2015) 12(3):255-67. doi:10.1080/15476286.2015.1017221

23. Asano N, Kato K, Nakamura A, Komoda K, Tanaka I, Yao M. Structural and functional analysis of the Rpf2-Rrs1 complex in ribosome biogenesis. Nucleic Acids Res (2015) 43(9):4746-57. doi:10.1093/nar/gkv305

24. Assoun S, Brosseau S, Steinmetz C, Gounant V, Zalcman G. Bevacizumab in advanced lung cancer: state of the art. Future Oncol (2017) 13(28):2515-35. doi:10.2217/fon-2017-0302

25. Knispel S, Zimmer L, Kanaki T, Ugurel S, Schadendorf D, Livingstone E. The safety and efficacy of dabrafenib and trametinib for the treatment of melanoma. Expert Opin Drug Saf (2018) 17(1):73-87. doi:10.1080/ 14740338.2018.1390562

26. Fornasier G, Francescon S, Baldo P. An update of efficacy and safety of cetuximab in metastatic colorectal cancer: a narrative review. Adv Ther (2018) 35(10):1497-509. doi:10.1007/s12325-018-0791-0 
27. Shields S, Conroy E, O'Grady T, McGoldrick A, Connor K, Ward MP, et al. BAG3 promotes tumour cell proliferation by regulating EGFR signal transduction pathways in triple negative breast cancer. Oncotarget (2018) 9(21):15673-90. doi:10.18632/oncotarget.24590

28. Lv J, Zhang F, Zhai C, Wang G, Qu Y. Bag-1 silence sensitizes non-small cell lung cancer cells to cisplatin through multiple gene pathways. Ott (2019) 12: 8977-89. doi:10.2147/ott.s218182

29. Kilbas PO, Akcay IM, Doganay GD, Arisan ED. Bag-1 silencing enhanced chemotherapeutic drug-induced apoptosis in MCF-7 breast cancer cells affecting PI3K/Akt/mTOR and MAPK signaling pathways. Mol Biol Rep (2019) 46(1):847-60. doi:10.1007/s11033-018-4540-x

30. Kizilboga T, Baskale EA, Yildiz J, Akcay IM, Zemheri E, Can ND, et al. Bag-1 stimulates bad phosphorylation through activation of Akt and Raf kinases to mediate cell survival in breast cancer. BMC Cancer (2019) 19(1):1254. doi:10. 1186/s12885-019-6477-4

31. Bruchmann A, Roller C, Walther TV, Schäfer G, Lehmusvaara S, Visakorpi T, et al. Bcl-2 associated athanogene 5 (Bag5) is overexpressed in prostate cancer and inhibits ER-stress induced apoptosis. BMC Cancer (2013) 13:96. doi:10.1186/1471-2407-13-96

32. Ni W, Chen B, Zhou G, Lu C, Xiao M, Guan C, et al. Overexpressed nuclear BAG-1 in human hepatocellular carcinoma is associated with poor prognosis and resistance to doxorubicin. J Cel Biochem. (2013) 114(9):2120-30. doi:10. $1002 /$ jcb. 24560

33. Kong D-H, Li S, Du Z-X, Liu C, Liu B-Q, Li C, et al. BAG3 elevation inhibits cell proliferation via direct interaction with G6PD in hepatocellular carcinomas. Oncotarget (2016) 7(1):700-11. doi:10.18632/oncotarget.6396

34. Xiao H, Cheng S, Tong R, Lv Z, Ding C, Du C, et al. BAG3 regulates epithelialmesenchymal transition and angiogenesis in human hepatocellular carcinoma. Lab Invest (2014) 94(3):252-61. doi:10.1038/labinvest.2013.151

35. Catez F, Dalla Venezia N, Marcel V, Zorbas C, Lafontaine DLJ, Diaz J-J. Ribosome biogenesis: an emerging druggable pathway for cancer therapeutics. Biochem Pharmacol (2019) 159:74-81. doi:10.1016/j.bcp.2018.11.014

36. Derenzini M, Montanaro L, Trerè D. Ribosome biogenesis and cancer. Acta Histochem (2017) 119(3):190-7. doi:10.1016/j.acthis.2017.01.009

37. Sharifi S, Bierhoff $\mathrm{H}$. Regulation of RNA polymerase I transcription in development, disease, and aging. Annu Rev Biochem (2018) 87:51-73. doi:10.1146/annurev-biochem-062917-012612

38. Ahuja R, Kumar V. Stimulation of Pol III-dependent $5 \mathrm{~S}$ rRNA and U6 snRNA gene expression by AP-1 transcription factors. FEBS J (2017) 284(13):2066-77. doi:10.1111/febs.14104

39. Liao P, Zeng SX, Zhou X, Chen T, Zhou F, Cao B, et al. Mutant $\mathrm{p} 53$ gains its function via c-myc activation upon CDK4 phosphorylation at serine 249 and consequent PIN1 binding. $\mathrm{Mol} \mathrm{Cel} \mathrm{(2017)} \mathrm{68(6):1134-46.} \mathrm{doi:10.1016/.molcel.2017.11.006}$

40. Calderaro J, Couchy G, Imbeaud S, Amaddeo G, Letouzé E, Blanc J-F, et al. Histological subtypes of hepatocellular carcinoma are related to gene mutations and molecular tumour classification. J Hepatol (2017) 67(4): 727-38. doi:10.1016/j.jhep.2017.05.014

41. Li Q, Liu X, Jin K, Lu M, Zhang C, Du X, et al. NAT10 is upregulated in hepatocellular carcinoma and enhances mutant p53 activity. BMC Cancer (2017) 17(1):605. doi:10.1186/s12885-017-3570-4

42. Piazzi M, Bavelloni A, Gallo A, Faenza I, Blalock WL. Signal transduction in ribosome biogenesis: a recipe to avoid disaster. Int J Mol Sci (2019) 20(11): 2718. doi:10.3390/ijms20112718
43. Qin L, Guo J, Zheng Q, Zhang H. BAG2 structure, function and involvement in disease. Cell Mol Biol Lett (2016) 21:18. doi:10.1186/s11658-016-0020-2

44. Komarova EY, Afanasyeva EA, Bulatova MM, Cheetham ME, Margulis BA, Guzhova IV. Downstream caspases are novel targets for the antiapoptotic activity of the molecular chaperone hsp70. Cell Stress Chaper (2004) 9(3): 265-75. doi:10.1379/csc-27r1.1

45. Sverchinsky DV, Nikotina AD, Komarova EY, Mikhaylova ER, Aksenov ND, Lazarev VF, et al. Etoposide-induced apoptosis in cancer cells can be reinforced by an uncoupled link between Hsp70 and caspase-3. Int J Mol Sci (2018) 19(9): 2519. doi:10.3390/ijms19092519

46. Kroemer G. Heat shock protein 70 neutralizes apoptosis-inducing factor. The Scientific World JOURNAL (2001) 1:590-2. doi:10.1100/tsw.2001.322

47. Gotoh T, Terada K, Oyadomari S, Mori M. hsp70-DnaJ chaperone pair prevents nitric oxide- and CHOP-induced apoptosis by inhibiting translocation of Bax to mitochondria. Cel Death Differ (2004) 11(4): 390-402. doi:10.1038/sj.cdd.4401369

48. Saleh A, Srinivasula SM, Balkir L, Robbins PD, Alnemri ES. Negative regulation of the apaf-1 apoptosome by Hsp70. Nat Cel Biol (2000) 2(8): 476-83. doi:10.1038/35019510

49. Bruckheimer EM, Cho SH, Sarkiss M, Herrmann J, McDonnell TJ. The Bcl-2 gene family and apoptosis. Adv Biochem Eng Biotechnol (1998) 62:75-105. doi:10.1007/bfb0102306

50. Nadler Y, Camp RL, Giltnane JM, Moeder C, Rimm DL, Kluger HM, et al. Expression patterns and prognostic value of Bag- 1 and Bcl-2 in breast cancer. Breast Cancer Res (2008) 10(2):R35. doi:10.1186/bcr1998

51. Xue C, Wang K, Jiang X, Gu C, Yu G, Zhong Y, et al. The down-regulation of SUZ12 accelerates the migration and invasion of liver cancer cells via activating ERK1/2 pathway. J Cancer (2019) 10(6):1375-84. doi:10.7150/jca. 29932

52. Gao X, Shan W, Liu X, Zhang J, Zheng J, Yao H. JNK1/2 and ERK1/2 provides vital clues about tumor recurrence and survival in hepatocellular carcinoma patients. Future Oncol (2018) 14(24):2471-81. doi:10.2217/ fon-2018-0171

53. Rhee H, Kim H-Y, Choi J-H, Woo HG, Yoo JE, Nahm JH, et al. Keratin 19 expression in hepatocellular carcinoma is regulated by fibroblast-derived HGF via a MET-ERK1/2-AP1 and SP1 axis. Cancer Res (2018) 78(7):1619-31. doi:10.1158/0008-5472.can-17-0988

54. Xu Z-Z, Xiu P, Lv J-W, Wang F-H, Dong X-F, Liu F, et al. Integrin $a v \beta 3$ is required for cathepsin B-induced hepatocellular carcinoma progression. $\mathrm{Mol}$ Med Rep (2015) 11(5):3499-504. doi:10.3892/mmr.2014.3140

55. Varshosaz J, Fard MM, Mirian M, Hassanzadeh F. Targeted nanoparticles for Co-delivery of 5-FU and nitroxoline, a cathepsin B inhibitor, in HepG2 cells of hepatocellular carcinoma. Anticancer Agents Med Chem (2020) 20(3):346-358. doi:10.2174/1871520619666190930124746

Copyright $\odot 2021$ Zhang, Zhang, Liu, Li, Tan and Song. This is an open-access article distributed under the terms of the Creative Commons Attribution License (CC $B Y)$. The use, distribution or reproduction in other forums is permitted, provided the original author(s) and the copyright owner(s) are credited and that the original publication in this journal is cited, in accordance with accepted academic practice. No use, distribution or reproduction is permitted which does not comply with these terms. 